Aim of the study: Our aim was to determine the activity and toxicity of uracil/tegafur and leucovorin combination in metastatic colorectal cancer (mCRC) patients who have progressed with all currently active agents.

Material and methods: This study was a retrospective analysis of $50 \mathrm{mCRC}$ patients who had previously failed to respond to all available chemotherapeutics and who received subsequent treatment with uracil/tegafur $250 \mathrm{mg} / \mathrm{m}^{2}$ d1-5 in combination with leucovorin $90 \mathrm{mg} /$ day, d1-5 followed by two days' rest.

Results: The median age of the patients was 60 years. Most of them $(60 \%)$ were male. Bevacizumab was used in $65 \%$ and cetuximab in $55 \%$ of the patients. Thirty-nine patients $(78 \%)$ were treated with uracil/tegafur in the fourth line setting. The median treatment duration was 4.2 months (range, 2-24 months). The objective response rate and the disease control rate were $4 \%$ and $34 \%$, respectively. Median progression-free survival was 4.1 months ( $95 \% \mathrm{Cl}, 3.6-4.6$ months) and overall survival was 6.6 months (95\% Cl, $4.5-8.6$ months). Grade 3 or 4 toxicity was seen in $20 \%(n=10)$ of the patients while $60 \%(n=6)$ of them required dose reductions.

Conclusions: This retrospective data show that uracil/tegafur may be considered in heavily pretreated $\mathrm{mCRC}$ patients because of its activity, lower toxicity, and feasibility.

Key words: uracil/tegafur, metastatic colorectal cancer, heavily pretreated, salvage therapy.

Contemp Oncol (Pozn) 2015; 19: 385-390 DOI: $10.5114 /$ wo.2015.53374

\section{Uracil/tegafur as a possible salvage therapy in chemo-refractory colorectal cancer patients: a single institutional retrospective study}

\author{
İbrahim V. Bayoglu, Ibrahim Yildiz, Umut Varol, Suna Cokmert, \\ Ahmet Alacacioğlu, Yuksel Kucukzeybek, Murat Akyol, Lutfiye Demir, \\ Ahmet Dirican, Oktay Tarhan
}

Department of Medical Oncology, Izmir Katip Celebi University Ataturk, Training and Research Hospital, Turkey

\section{Introduction}

Colorectal cancer is one of the most important global health problems. Approximately $20 \%$ of colorectal cancer patients are diagnosed in the metastatic stage, and $50 \%$ of patients with CRC subsequently develop locally recurrent or distant metastatic disease following diagnosis [1, 2].

5-Fluorouracil (5-FU)-based chemotherapy has been the mainstay of treatment for metastatic colorectal cancer (mCRC). Leucovorin (LV), also known as folinic acid, can augment the therapeutic effects of 5-FU by enhancing the inhibition of DNA synthesis [3, 4]. So, leucovorin in combination with 5 -FU has received widespread acceptance as therapy for advanced CRC. Uracil/tegafur is an oral derivative of 5-FU that acts as a prodrug [5]. Tegafur is subsequently metabolised to 5-FU, and uracil is naturally metabolised by dihydropyrimidine dehydrogenase (DPD), so uracil significantly prolongs the half-life of 5-FU by competitive inhibition of the enzyme [6-10]. In addition, instead of the healthy tissues, this inhibition occurs especially in tumour cells as a result of increasing 5 -FU concentration and its antineoplastic activity. Also, when we combine leucovorin with UFT, it potentiates the effect of UFT on tumour cells in the same way as its administration with intravenous 5-FU.

5-Fluorouracil infusion or uracil/tegafur in combination with irinotecan and oxaliplatin has shown a good activity and tolerability profile in $\mathrm{mCRC}$ [11-14]. In the treatment of $\mathrm{mCRC}$ patients, both irinotecan and oxaliplatin have also been used in combination with UFT, with favourable anti-tumour activity. Recently, the vascular endothelial growth factor (VEGF) antibody bevacizumab has been shown to significantly improve the prognosis of mCRC when added to chemotherapy in the first- and second-line setting [15-17]. The epidermal growth factor receptor (EGFR) antibodies cetuximab and panitumumab have also been determined to be active in the treatment of irinotecan- and oxaliplatin-refractory $\mathrm{mCRC}$, especially in the wild-type K-ras population [18-21].

There is no standard therapy for heavily pretreated patients with good performance status. In a recent study, a novel oral multikinase inhibitor regorafenib improved overall survival when compared with best supportive care, in patients with $\mathrm{mCRC}$ who had received all available standard therapies [22]. According to this trial, survival is short without treatment, and these patients might be candidates for further therapy although the chemotherapeutic option for them is quite limited. Our aim in this study was to show the efficacy and toxicity of uracil/tegafur and leucovorin combination therapy in patients with $\mathrm{MCRC}$, who have progressed to all currently approved standard treatments. 


\section{Material and methods}

\section{Patient eligibility criteria}

We retrospectively reviewed the medical records of 56 metastatic colorectal patients who had received all currently approved standard therapies and who were treated with uracil/tegafur as a salvage therapy between July 2006 and June 2013. Available standard therapies in our country were fluoropyrimidine, oxaliplatin, irinotecan, bevacizum$a b$, and cetuximab. Our inclusion criteria were as follows: patients > 18 years old; Eastern Cooperative Oncology Group (ECOG) [23] PS of 0 to 2 and adequate haematological/clotting, hepatic, and renal function. Of these 56 patients, 4 were not available for evaluation because of the short duration of treatment ( $<4$ weeks), loss to follow-up, or rejection of further treatment. Two patients that had undergone metastasectomy were also excluded. Clinical data with complete information were available for 50 patients.

\section{Treatment and dose modifications}

Uracil/tegafur $250 \mathrm{mg} / \mathrm{m}^{2} /$ day and LV $90 \mathrm{mg}$ /day were given together, in three divided daily doses, for 5 days (days 1-5), followed by a 2-day rest period. In patients aged $>65$ years or with ECOG PS of 2 and having signifi-

Table 1. Characteristics of the patients

\begin{tabular}{|c|c|}
\hline Characteristic & No. of patients (\%) \\
\hline \multicolumn{2}{|l|}{ Sex } \\
\hline male & $30(40)$ \\
\hline female & $20(60)$ \\
\hline Median age (range) [years] & $60(37-76)$ \\
\hline \multicolumn{2}{|l|}{ ECOG performance status } \\
\hline 0 & $8(16)$ \\
\hline 1 & $26(52)$ \\
\hline 2 & $16(32)$ \\
\hline \multicolumn{2}{|l|}{ Primary site } \\
\hline colon & $28(56)$ \\
\hline rectum & $14(28)$ \\
\hline colorectal & $8(16)$ \\
\hline \multicolumn{2}{|l|}{ Number of organs involved } \\
\hline 1 & $18(26)$ \\
\hline 2 & $20(40)$ \\
\hline$\geq 3$ & $12(24)$ \\
\hline \multicolumn{2}{|l|}{ Organ involvement } \\
\hline liver & $36(72)$ \\
\hline lung & $26(52)$ \\
\hline lymph nodes & $9(18)$ \\
\hline peritoneum & $4(8)$ \\
\hline bone & $3(6)$ \\
\hline \multicolumn{2}{|l|}{ KRAS status } \\
\hline mutated & $10(20)$ \\
\hline wild & $18(36)$ \\
\hline unknown & $22(44)$ \\
\hline
\end{tabular}

ECOG - Eastern Cooperative Oncology Group cant co-morbidities, the treatment was started with a $25 \%$ dose reduction. After a two-week treatment period, for patients without grade 3 or 4 toxicity or intolerance to the treatment, the dose of the drug was increased to its standard dosage.

\section{Response evaluation}

Response Evaluation Criteria in Solid Tumours (RECIST 1.1) [24] criteria were applied for tumour measurement. Haematological and non-haematological toxic effects were graded according to the Common Terminology Criteria for Adverse Events v3.0 (CTCAE v3.0) [25]. Response evaluation was performed during and after the treatment at every eight weeks by laboratory studies, computed tomography $(\mathrm{CT})$, or magnetic resonance imaging (MRI). Treatment was administered until the disease progression, unacceptable toxicity, or rejection of treatment continuation. Progression-free survival (PFS) was calculated as the time from the first day of treatment to the first day of documented progression or death. We censored the last clinical visit data for patients who died without known progression.

\section{Statistical analysis}

Quantitative data are presented as the means, medians, minimums, and maximums, whereas the results of qualitative analyses are presented as frequencies and percentages. Survival curves were estimated using the Kaplan-Meier method and the log-rank test was used to compare curves. All P-values represent two-sided tests of statistical significance. P-values $<0.05$ were considered statistically significant. SPSS version 16.0 (SPSS Inc., Chicago, IL) was used for the statistical analyses.

\section{Results}

\section{Patient characteristics and treatment details}

Among the fifty patients, most of them ( $\mathrm{n}: 28 ; 56 \%$ ) were colon cancer, 14 (28\%) were rectal cancer, and 8 (16\%) were colorectal cancer patients. The sample comprised 30 males (60\%) and 20 females (40\%). Median age of the patients was 60 years (range, 37-76 years). Thirty-four patients (68\%) had an ECOG PS of 0 to 1 , while the rest had PS of 2 (32\%). Patient demographics are listed in Table 1.

All of the patients had received prior 5-FU, irinotecanand oxaliplatin-based chemotherapy regimens for their metastatic disease. Bevacizumab and cetuximab was used in 42 (84\%) and 40 (80\%) patients, respectively. Bevacizumab was not used in eight patients because of the contraindication for treatment. Ten patients had K-RAS mutation, 18 had wild type K-RAS, and 22 patients' K-RAS status was unknown. For this reason, 10 (20\%) patients with mutant K-RAS had uracil/tegafur treatment in thirdline and 40 (80\%) patients were treated in the forth-line setting with uracil/tegafur. Treatment details of the patients are shown in Table 2. 
Table 2. Treatment characteristics and efficacy of uracil/tegafur

\begin{tabular}{lc} 
Initial treatment & $n(\%)$ \\
adjuvant-neoadjuvant chemotherapy & \\
adjuvant radiotherapy & $23(46)$ \\
diagnosed at metastatic stage & $8(16)$ \\
Surgical treatment & $32(64)$ \\
\hline undergone surgical resection & \\
palliative surgical procedures & $29(58)$ \\
the median DFS for operated patients & $6(12)$ \\
(range) [months] & $13(4.9-56)$ \\
Response to uracil/tegafur & \\
complete response & \\
partial response & \\
stable disease & $0(0)$ \\
progression & $2(4)$ \\
uracil/tegafur responders & $15(30)$ \\
uracil/tegafur refractory & $33(66)$ \\
Median PFS, months (95\% CI) & $17(34)$ \\
Median OS, months (95\% CI) & $33(66)$ \\
for all patients & $4.1(3.6-4.6)$ \\
uracil/tegafur responders & \\
uracil/tegafur refractory & \\
3 ld line treatment with uracil/tegafur & $6.6(4.5-8.6)$ \\
$4^{\text {th }}$ line treatment with uracil/tegafur & $7.7(4.1-11)$ \\
DFS - disease-free survival; PFS - progression-free survival; OS - overall \\
survival
\end{tabular}

\section{Clinical outcome and toxicity}

The median treatment duration for uracil/tegafur therapy was 4.2 months (range, 2-24 months) and treatment was ongoing in three patients (6\%) at the time of analysis. Seventeen (34\%) patients had disease control with chemotherapy. Only two patients (4\%) had partial response to uracil/tegafur treatment and 15 patients (30\%) had stable disease. No complete response was observed (Table 2). For all patients, median PFS was 4.1 months $(95 \% \mathrm{Cl}, 3.6-4.6$ months; Fig. 1) and median OS was 6.6 months $(95 \% \mathrm{Cl}$, 4.5-8.6 months; Fig. 2). In univariate analysis with respect to initial staging (local vs. metastatic), primary tumour resection (present vs. not), organ involved like lung, liver, or peritoneal (present vs. not), sex (male vs. female), and age (> 60 vs. < 60) there was no correlation between these demographic parameters and median OS of the patients treated with uracil/tegafur therapy.

There was a limited number of patients ( $\mathrm{n}$ : 12; both for first cycle of chemotherapy due to ECOG 2 with comorbidities and for other chemotherapy cycles after grade 3 or 4 toxicity) whose dose intensity for uracil/tegafur was reduced. In addition, during the follow-up, most of these patients had received the planned dosages of UFT. When we compared the survival outcome with respect to that, we found the median OS of the patients who had reduced dose intensity of UFT was 6.2 months $(95 \% \mathrm{Cl}, 2.1-10.1$ months) whereas median OS of the patients who had

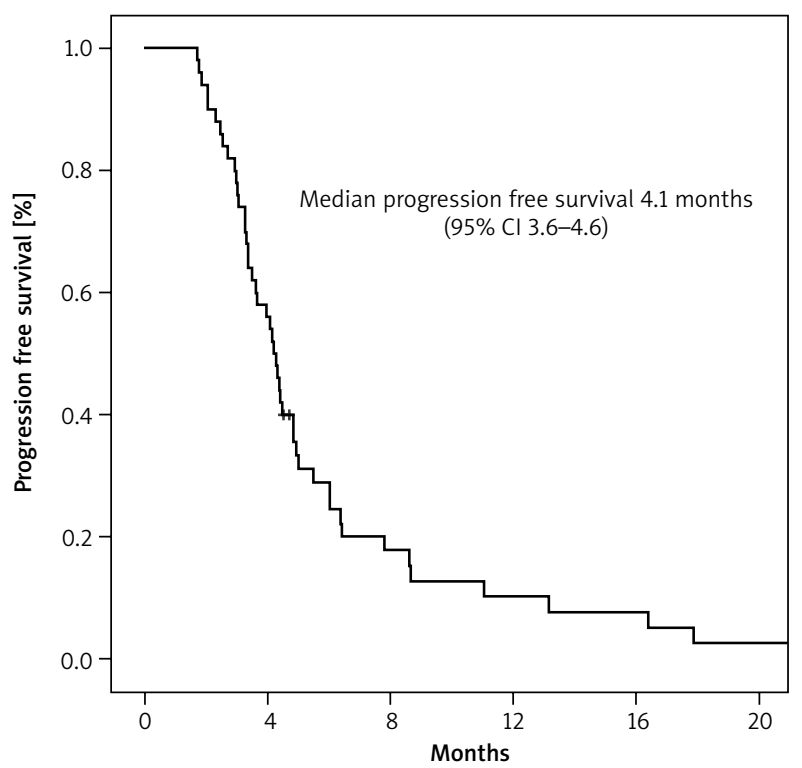

Fig. 1. Median progression-free survival of uracil/tegafur treatment

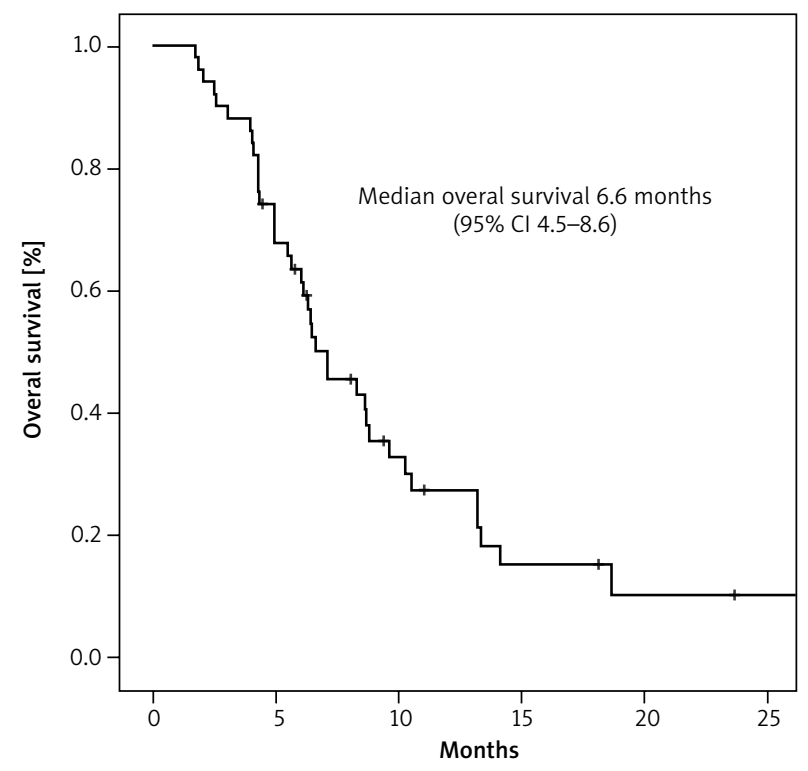

Fig. 2. Median overall survival of uracil/tegafur treatment

standard dosage of UFT was 6.6 months $(95 \% \mathrm{Cl}$, 4.2-9.0 months) $(p=0.44)$.

When we analysed the OS time for all patients, from the start of first-line treatment, we found it to be 30.8 months (95\% Cl, 27.1-34.5; Fig. 3). According to their response to uracil/tegafur treatment, median survival of uracil/tegafur responders (patients with a partial response or stable disease; n: 17) was 7.7 months $(95 \% \mathrm{Cl}, 4.1-11)$ compared with 3.4 months (95\% Cl, 2.9-3.7; Fig. 4) for uracil/tegafur refractory (patients with progressive disease; $n=33$ ) patients. There was a statistically significant difference between these two groups $(p<0.001)$.

The most common reason for treatment withdrawal was disease progression (n: $33 ; 66 \%$ ). Grade 3 or 4 toxicity was seen in 20\% (n: 10) of the patients while 60\% (n: 6 ) of them required dose reductions. There was no death among 


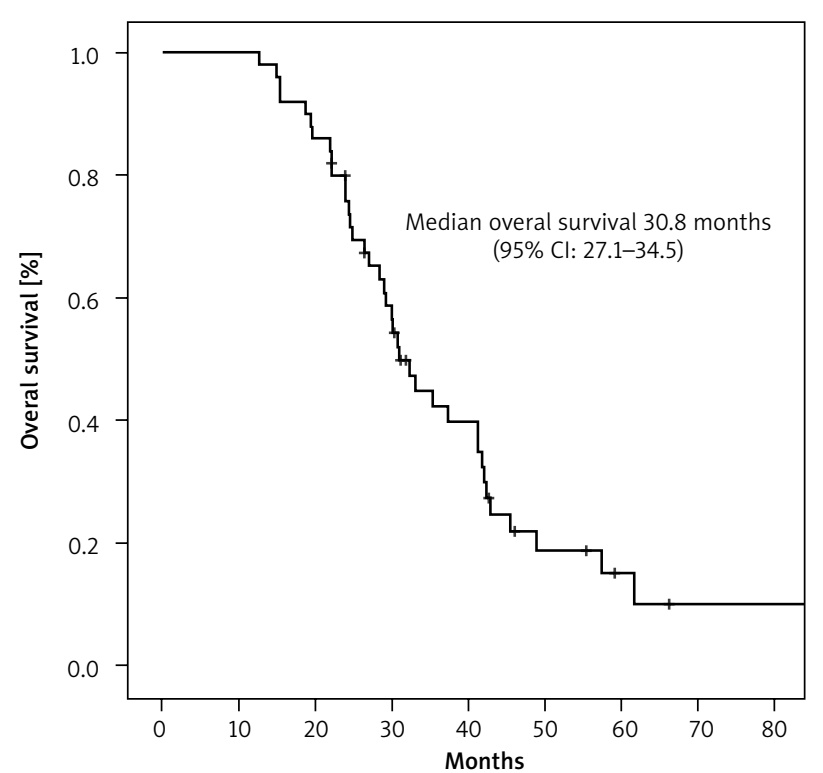

Fig. 3. Median overall survival of the patients following all of the treatment

Table 3. Toxicity of uracil/tegafur

\begin{tabular}{|c|c|c|}
\hline \multirow[t]{2}{*}{ Toxicity } & \multicolumn{2}{|c|}{ Grade } \\
\hline & $1-2$ & $3-4$ \\
\hline \multicolumn{3}{|l|}{ Hematologic toxicity } \\
\hline anaemia & $9(18 \%)$ & $2(4 \%)$ \\
\hline neutropenia & $7(14 \%)$ & $2(6 \%)$ \\
\hline thrombocytopenia & $6(12 \%)$ & - \\
\hline \multicolumn{3}{|l|}{ Non-hematologic toxicity } \\
\hline diarrhoea & $14(28 \%)$ & $4(8 \%)$ \\
\hline nausea & $11(22 \%)$ & $1(2 \%)$ \\
\hline vomiting & $5(10 \%)$ & $1(2 \%)$ \\
\hline mucositis & $5(10 \%)$ & $2(4 \%)$ \\
\hline fatigue & $14(28 \%)$ & $7(14 \%)$ \\
\hline hand-foot send & $2(4 \%)$ & - \\
\hline neuropathy & $4(8 \%)$ & $1(2 \%)$ \\
\hline
\end{tabular}

our patients related with uracil/tegafur and no patient was hospitalised because of grade 3 or 4 toxicities. The most common grade 3 or 4 toxicities were fatigue (14\%). Table 3 lists the common treatment-related toxicities.

\section{Discussion}

In our study, the patients were heavily pretreated with a median of three major chemotherapy regimens for mCRC. Nevertheless, we found that the patients treated with uracil/tegafur had median PFS of 4.1 months and median OS of 6.6 months, respectively. The toxicity profile of the treatment was manageable and uracil/tegafur shows potential activity as a salvage therapy in $\mathrm{MCRC}$ patients who were treated with all approved standard therapies.

5-Fluorouracil is the cornerstone of treatment in the management of $\mathrm{mCRC}$ [26]. During the last two decades combinations of 5-FU with irinotecan, oxaliplatin, and bevacizum-

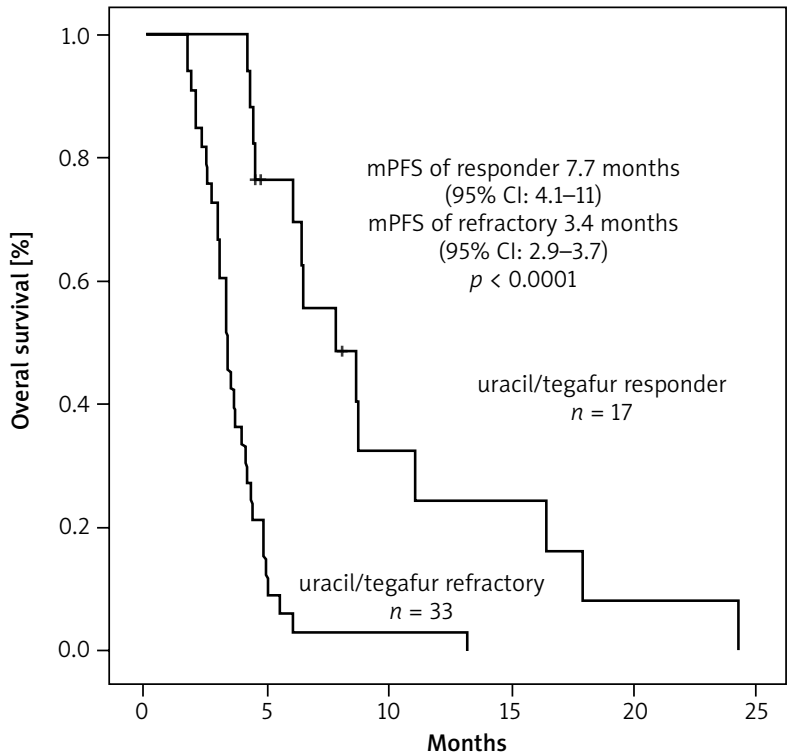

Fig. 4. Overall survival curves for uracil/tegafur responder and refractory patients

ab have proven effective for first- and as well as second-line treatment of $m C R C[15,16]$. In addition, recent studies have shown that treatment with cetuximab and panitumumab can improve survival in K-RAS wild type tumours [18-21]. The median OS has been extended to over 20 months when all available agents are administered during the course of the disease [27]. However, further treatment of patients who have progressed after all these agents and have good performance status remain unclear. With best supportive care only, median PFS of two months and OS of five months were reported for those patients [22].

Uracil/tegafur was generally found to be well tolerated and effective in patients with $\mathrm{mCRC}[14,28]$. However, the activity of uracil/tegafur in chemotherapy refractory patients is not clear. Besides, for heavily pretreated patients, there have been few reports about salvage therapy. The rationale for using uracil/tegafur in refractory patients is due to increased antineoplastic activity in tumour cells instead of healthy tissue as a result of increasing intratumoural 5-FU concentration. In patients pretreated with 5FU, irinotecan, and oxaliplatin, disease control rates and survivals were $33.6 \%$ and 5 months with raltitrexed plus mitomycin-C, $15 \%$ and 6.1 months with capecitabine, and $23.8 \%$ and 6.8 months with capecitabine plus mitomycin-C [29-31]. Single-agent capecitabine has recently been tried as a third- or even fourth-line treatment and has demonstrated a median TTP of 4 months and median OS of 5.2 months [32]. These results were similar to those from our study but with more frequent grade 3 or 4 toxicity, such as hand-foot syndrome (21\%), diarrhoea (25\%), and nausea (29\%), which was observed with capecitabine. Another oral 5-FU derivative S-1 treatment showed a response rate of $15 \%$ in irinotecan- and oxaliplatin-resistant mCRC patients. Four patients who obtained objective tumour response had median response duration of 200 days, suggesting that antitumor activity of S-1 was durable even in heavily pretreated patients once response was induced. So, S-1 treatment showed efficiency 
with favourable safety profiles even in a refractory setting [33]. Similar to S1, our results demonstrated that median OS and PFS were significantly superior in uracil/tegafur responders than in refractory patients.

Larsen et al. evaluated the combination of capecitabine and bevacizumab in heavily pre-treated $\mathrm{MCRC}$ patients, and this combination has resulted in a median PFS of 5.4 months and median OS of 12.2 months [34]. In a recent study Vincenzi et al. determined the efficacy and safety of bevacizumab and 5-FU/folinic acid in patients who had received all other anticancer drugs available for the treatment of $\mathrm{mCRC}$ [35]. Median TTP was found to be four months and median OS was found nine months. However, 21 (43\%) patients required treatment delay or dose reduction. The median TTP was found to be 2 months and median OS was found to be 4.5 months in patients who required treatment delay or dose reduction.

Regorafenib, a novel target agent, was evaluated in a phase III CORRECT trial in patients with advanced or metastatic CRC, who had received all currently approved standard therapies [22, 36]. The addition of regorafenib to best supportive care increased OS (6.4 vs. 5.0 months) and PFS (1.9 months vs. 1.7 months) when compared with best supportive care alone. However, although small-molecule tyrosine kinase inhibitor regorafenib has shown little but significant OS benefit in patients with treatment refractory $\mathrm{mCRC}$, the high cost of the treatment can lead to a considerable financial burden, and the toxicity of the drug can raise some concern $(51 \%$ and $3 \%$ of the patients experienced grade 3 and 4 adverse events, respectively). On the other hand, despite the huge difference between regorafenib and uracil/tegafur in terms of treatment costs, both drugs seemed to have similar tolerability.

Grade 3/4 haematological and non-haematological adverse events were uncommon in the present study. The most frequent grade $3 / 4$ treatment-related adverse effects were neutropenia (6\%), diarrhoea (8\%) and fatigue (14\%). However, grade $1 / 2$ diarrhoea occurred in the majority $(28 \%)$ of patients, indicating that it was effectively managed in most patients by supportive measures and anti-diarrheal medications. Hand-foot syndrome, which is a frequent side effect of infusional 5-FU and capecitabine, was never observed in our study. Frequent oral uracil/tegafur/LV dosing in our patients may be the reason for the absence of hand-foot syndrome.

In conclusion, despite its methodological limitations and small sample size, our study clearly indicates that salvage treatment with single-agent uracil/tegafur may be considered in heavily pretreated $\mathrm{MCRC}$ patients because of its cost-effectiveness, safety profile, and feasibility.

\section{The authors declare no conflict of interest.}

\section{References}

1. Siegel R, Naishadham D, Jemal A. Cancer statistics, 2013. CA Cancer J Clin 2013; 63: 11-30.

2. Van Cutsem E, Nordlinger B, Cervantes A. Advanced colorectal cancer: ESMO clinical practice guidelines for treatment. Ann Oncol 2010; 21: 93-7.
3. Tsai CM, Gazdar AF, Allegra C, Perng RP, Kramer BS. Enhancement of fluorinated pyrimidine-induced cytotoxicity by leucovorin in human lung cancer cell lines. Int J Cancer 1990; 46: 101-5.

4. Thirion P, Michiels S, Pignon JP, et al. Modulation of fluorouracil by leucovorin in patients with advanced colorectal cancer: an updated meta-analysis. J Clin Oncol 2004; 22: 3766-75. Erratum in: J Clin Oncol 2005; 23: 1337-8.

5. El Sayed YM, Sadee W. Metabolic activation of R,S-1-(tetrahydro-2-furanyl)-5-fluorouracil (ftorafur) to 5-fluorouracil by soluble enzymes. Cancer Res 1983; 43: 4039-44.

6. Ikenaka T, Shiraska T, Kitano S, Fujii S. Effect of uracil on metabolism of 5-fluorouracil in vitro. Gann 1979; 70: 353-9.

7. Hoff PM, Pazdur R, Benner SE, Canetta R. UFT and leucovorin: a review of its clinical development and therapeutic potential in the oral treatment of cancer. Anticancer Drugs 1998; 9: 479-90.

8. Tsujimoto T, Sakai S, Murata M. Concentrations of 5-FU in the tissue and serum of patients with head and neck malignant tumor by preoperative administration of UFT. Jpn I Cancer Chemother 1983; 10: 78-83.

9. Sanchiz F, Milla A. Tegafur-uracil (UFT) plus leucovorin in advanced rectal cancer. Jpn J Clin Oncol 1994; 24: 322-6.

10. Pazdur R, Lassere $Y$, Rhodes V, et al. Phase II trial of uracil and tegafur plus oral leucovorin: an effective oral regimen in the treatment of metastatic colorectal carcinoma. J Clin Oncol 1994; 12: 2296-300.

11. Saltz LB, Cox JV, Blanke C, et al. Irinotecan plus fluorouracil and leucovorin for metastatic colorectal cancer. Irinotecan Study Group. N Engl J Med 2000; 343: 905-14.

12. Goldberg RM, Sargent DJ, Morton RF, et al. A randomized controlled trial of fluorouracil plus leucovorin, irinotecan, and oxaliplatin combinations in patients with previously untreated metastatic colorectal cancer. J Clin Oncol 2004; 22: 23-30.

13. Tournigand C, André T, Achille E, Lledo G, Flesh M, Mery-Mignard D, et al. FOLFIRI followed by FOLFOX6 or the reverse sequence in advanced colorectal cancer: a randomized GERCOR study. I Clin Oncol 2004; 22: 229-37.

14. Bajetta E, Di Bartolomeo M, Buzzoni R, et al. Uracil/ftorafur/leucovorin combined with irinotecan (TEGAFIRI) or oxaliplatin (TEGAFOX) as first-line treatment for metastatic colorectal cancer patients: results of randomised phase II study. Br J Cancer 2007; 96: 439-44.

15. Hurwitz H, Fehrenbacher L, Novotny W, et al. Bevacizumab plus irinotecan, fluorouracil, and leucovorin for metastatic colorectal cancer. N Engl J Med 2004; 350: 2335-42.

16. Giantonio BJ, Catalano PJ, Meropol NJ, et al. Bevacizumab in combination with oxaliplatin, fluorouracil, and leucovorin (FOLFOX4) for previously treated metastatic colorectal cancer: results from the Eastern Cooperative Oncology Group Study E3200. J Clin Oncol 2007; 25: 1539-44.

17. Van Cutsem E, Rivera F, Berry S, et al. First BEAT investigators. Safety and efficacy of first-line bevacizumab with FOLFOX, XELOX, FOLFIRI and fluoropyrimidines in metastatic colorectal cancer: the BEAT study. Ann Oncol 2009; 20: 1842-7.

18. Cunningham D, Humblet $Y$, Siena S, et al. Cetuximab monotherapy and cetuximab plus irinotecan in irinotecan-refractory metastatic colorectal cancer. N Engl J Med 2004; 351: 337-345.

19. Gibson TB, Ranganathan A, Grothey A. Randomized Phase III trial results of panitumumab, a fully human anti-epidermal growth factor receptor monoclonal antibody, in metastatic colorectal cancer. Clin Colorectal Cancer 2006; 6: 29-31.

20. Peeters M, Price TJ, Cervantes A, et al. Final results from a randomized phase 3 study of FOLFIRI $\{+/-\}$ panitumumab for second-line treatment of metastatic colorectal cancer. Ann Oncol 2014; 25: 107-16.

21. Van Cutsem E, Köhne CH, Hitre E, et al. Cetuximab and chemotherapy as initial treatment for metastatic colorectal cancer. N Engl J Med 2009; 360: 1408-17.

22. Grothey A, Van Cutsem E, Sobrero A, et al. CORRECT Study Group. Regorafenib monotherapy for previously treated metastatic colorectal cancer (CORRECT): an international, multicentre, randomised, placebo-controlled, phase 3 trial. Lancet 2013; 381: 303-12. 
23. Oken MM, Creech RH, Tormey DC, et al. Toxicity and response criteria of the Eastern Cooperative Oncology Group. Am J Clin Oncol 1982; 5: 649-55.

24. Eisenhauer EA, Therasse P, Bogaerts J, et al. New response evaluation criteria in solid tumours: revised RECIST guideline (version 1.1). Eur J Cancer 2009; 45: 228-47.

25. Trotti A, Colevas AD, Setser A, et al. CTCAE v3.0: development of a comprehensive grading system for the adverse effects of cancer treatment. Semin Radiat Oncol 2003; 13: 176-81.

26. Efficacy of intravenous continuous infusion of fluorouracil compared with bolus administration in advanced colorectal cancer. Meta-analysis Group In Cancer. J Clin Oncol 1998; 16: 301-18.

27. Gallagher DJ, Kemeny N. Metastatic colorectal cancer: from improved survival to potential cure. Oncology 2010; 78: 237-48.

28. Sheikh HY, Valle JW, Palmer K, et al. Concurrent irinotecan, oxaliplatin and UFT in firstline treatment of metastatic colorectal cancer: a phase I study. Br J Cancer 2007; 96: 38-43.

29. Lim do H, Park YS, Park BB, et al. Mitomycin-C and capecitabine as third-line chemotherapy in patients with advanced colorectal cancer: a phase II study. Cancer Chemother Pharmacol 2005; 56: 10-4.

30. Rosati G, Rossi A, Germano D, Reggiardo G, Manzione L. Raltitrexed and mitomycin- $C$ as third-line chemotherapy for colorectal cancer after combination regimens including 5-fluorouracil, irinotecan and oxaliplatin: a phase II study. Anticancer Res 2003; 23: 2981-5.

31. Gubanski M, Naucler G, Almerud A, Lideståhl A, Lind PA. Capecitabine as third line therapy in patients with advanced colorectal cancer. Acta Oncol 2005; 44: 236-9.

32. Ardavanis AS, Ioannidis GN, Orphanos GS, Rigatos GA. Salvage treatment with single-agent capecitabine in patients with heavily pretreated advanced colorectal cancer. Anticancer Res 2006; 26: 1669-72.

33. Jeung HC, Rha SY, Cho BC, et al. A phase II trial of S-1 monotherapy in metastatic colorectal cancer after failure of irinotecan- and oxaliplatin-containing regimens. Br J Cancer 2006; 95: 1637-41.

34. Larsen FO, Boisen MK, Fromm AL, Jensen BV. Capecitabine and bevacizumab in heavily pre-treated patients with advanced colorectal cancer. Acta Oncol 2012; 51: 231-3.

35. Vincenzi B, Santini D, Russo A, et al. Bevacizumab in association with de Gramont 5-fluorouracil/folinic acid in patients with oxaliplatin-irinotecan-and cetuximab-refractory colorectal cancer: a single-center phase 2 trial. Cancer 2009; 115: 4849-56.

36. Nielsen DL, Palshof JA, Larsen FO, Jensen BV, Pfeiffer P. A systematic review of salvage therapy to patients with metastatic colorectal cancer previously treated with fluorouracil, oxaliplatin and irinotecan +/- targeted therapy. Cancer Treat Rev 2014; 40: 701-15.

\section{Address for correspondence}

\section{Ahmet Alacacloğlu}

Department of Medical Oncology

Izir Katip Celebi Hospital and Medical Faculty

Department of Medical Oncology

35620 Izmir, Turkey

e-mail: dralaca2000@yahoo.com

Submitted: 7.03 .2014

Accepted: 29.05 .2014 